\title{
A tomographic test of cosmic anisotropy with the recently-released quasar sample
}

\author{
Dong Zhao, Jun-Qing Xia ${ }^{\mathrm{a}}$ \\ Department of Astronomy, Beijing Normal University, Beijing 100875, China
}

Received: 25 July 2021 / Accepted: 28 September 2021 / Published online: 26 October 2021

(C) The Author(s) 2021

\begin{abstract}
We test the cosmic anisotropy in the dipolemodulated $\Lambda \mathrm{CDM}$ model and Finslerian cosmological model with the recently-released quasar sample. Based on the redshift tomographic method, the quasar sample is divided into two subsets $z \leq z_{c u t}$ and $z>z_{\text {cut }}$ by different cutting redshifts. The dipole amplitudes of the two cosmological models from the subsets $z \leq z_{c u t}$ are very weak. We find that quasars at a higher redshift range may provide more detailed information about the dipole amplitude $A_{D}$. The dipole directions of each cosmological model from the subsets $z \leq 1.1$ and $z>1.1$ are deviated by $1 \sigma$ level. The Pantheon sample is combined with the two subsets. The dipole amplitude from the two combined datasets is also very weak. In the dipole-modulated $\Lambda \mathrm{CDM}$ model, the dipole direction from the combined dataset quasar at $z \leq 1.1$ and Pantheon sample is far away from the one given by the Pantheon sample. In the Finslerian cosmological model, the dipole directions from the two combined datasets are close to the one in the Pantheon sample.
\end{abstract}

\section{Introduction}

As one of the foundations in modern cosmology, the cosmological principle assumes that the Universe is homogeneous and isotropic at large scales [1]. The cosmological principle is well consistent with current cosmological observations, such as the Cosmic Microwave Background (CMB) radiation from the Wilkinson Microwave Anisotropy Probe (WMAP) [2,3] and Planck satellites [4-7]. Though the validity of the cosmological principle has been verified by the WMAP and Planck with high precision, there are still exist some phenomena which are inconsistent with the cosmological principle. An incomplete list includes the spatial variation of the electromagnetic fine-structure constant [8-11], the anisotropic accelerating expansion of the Universe [12-17], the MOND

a e-mail: xiajq@bnu.edu.cn (corresponding author) acceleration scale [18-20], the alignment of quasar polarization vectors [21-23], the alignment of quadrupole and octopole in the CMB temperature anisotropies [5,24-26], the hemispherical power asymmetry in CMB [27-30], the parity asymmetry in CMB [27,31-35], the large dipoles obtained in the number counts of radio and mid-IR sources [36-50], the anisotropies found in the $L_{X}-T$ relation in X-ray galaxy clusters [51-53], and the large-scale asymmetry of galaxy spin directions [54]. These phenomena hint that the existence of cosmic anisotropy.

The low redshift observational datasets used to test cosmological models are mostly in the redshift range $0 \leq z \leq 2.3$, such as the Type Ia supernova (SNe Ia) sample, the Hubble parameter observations, and the baryon acoustic oscillation measurements. The observational dataset at the higher redshift $z \sim 1100$ is cosmic microwave background anisotropy data from Planck and WMAP. Cosmological models are rarely tested in the intermediate redshift range $2.3 \leq z \leq$ 1100. An incomplete list of the datasets and related works to test cosmological models in the intermediate redshift range include the gamma-ray burst [55-66], the HIIG starburst galaxy [59,67-73], the quasar angular size [59,69,74-76], and the X-ray and UV flux of quasars [77-87].

In recent years, SNe Ia sample, i.e., the Union $2 \& 2.1$ sample [88,89], Joint Light-Curve Analysis (JLA) sample [90], and Pantheon sample [91] are widely used to test the anisotropy of the Universe. Certain preferred directions were found by the hemisphere comparison (HC) method with the Union2\&2.1 sample [15-17,92-98]. The $\Lambda$ CDM model is barely consistent with the SNe Ia data at the redshift range $z<0.5$ in the Union2 sample [99]. Mariano and Perivolaropoulos found a dipole direction at $2 \sigma$ level with the Union2 sample [10]. Zhao et al. found a dipole of cosmic acceleration at more than $2 \sigma$ level with the Union 2 sample [100]. Unlike the Union2\&2.1 sample, there is no anisotropic signal in the JLA sample [101-103] and Pantheon sample [104-106]. Zhao and Zhou et al. [107] found that the SDSS 
subsample has a decisive impact on the dipole anisotropy in the Pantheon sample.

Besides the SNe Ia sample, galaxy [18-20,54], galaxy cluster [51-53], number counts of radio and mid-IR sources [36-50], gamma-ray burst $[13,66,108]$, gravitational wave [109], fast radio burst $[109,110]$, and quasar $[83,87]$ are also used to investigate the cosmic anisotropy. The UV and $\mathrm{X}$-ray luminosity of quasars are used to test the cosmic anisotropy by Hu etc. [87]. They combined the 2019 compilation of quasars [78] with the Pantheon sample [91] to test the dipole-modulated $\Lambda \mathrm{CDM}$ model. They found that there is no evidence of cosmic anisotropy in the SN-Q sample. Zhao and Xia [83] tested the Finslerian cosmological model with the 2015 compilation of quasars. They found the cosmic anisotropy is very weak from the combination of the Pantheon sample and 2015 quasar compilation.

As the most luminous and persistent energy source, quasars have a lot of potential in exploring our Universe. Risaliti and Lusso (et al.) have been attempting to test cosmological models with the non-linear relation between the UV and X-ray luminosity of quasars since 2015 [77-79]. More recently, Lusso and Risaliti (et al.) released a new catalogue of optically selected quasars [79]. The 2020 compilation of quasars contains 2421 quasars that over the redshift range $0.009 \leq \mathrm{z} \leq 7.5413$. Due to the host-galaxy contamination, the $2500 \AA$ UV fluxes of quasars at the redshift range $z<0.7$ are less reliable. So the 2023 quasars at the redshift range $z>0.7$ and 15 local quasars with higher quality at the redshift range $z<0.7$ are used in the test of cosmic anisotropy.

In this work, we will analyze the quasar sample by using a redshift tomographic method. The quasar sample is divided into two subsets by different cutting redshifts. We will use the subsets to constrain the dipole-modulated $\Lambda \mathrm{CDM}$ model and Finslerian cosmological model. The Pantheon sample is combined with the subsets in our analysis. The rest of this paper is organized as follows. In Sect. 2, we briefly introduce the quasar sample and the two cosmological models. We show our results in Sect. 3. Finally, discussions and conclusions are given in Sect. 4.

\section{The quasar sample and methodology}

\subsection{The quasar sample}

The recently-released quasar compilation used in our work was presented by Lusso et al. [79]. The 2020 compilation consists of 2421 quasars over the redshift range $0.009 \leq \mathrm{z}$ $\leq 7.5413$. Because of the host-galaxy contamination at low redshift, the $2500 \AA$ UV fluxes of quasars at the redshift range $z<0.7$ are less reliable. We choose the quasars at the redshift range $z>0.7$ and 15 local quasars with higher quality at the

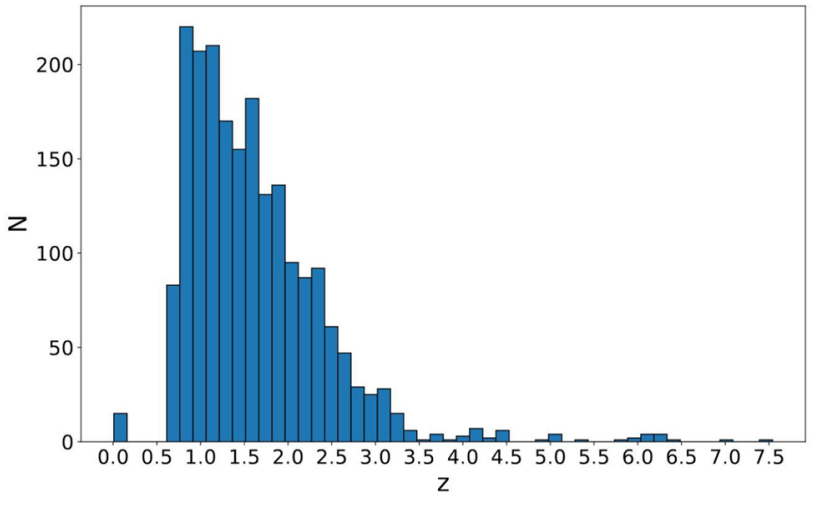

Fig. 1 The redshift distribution of the quasar sample

redshift range $z<0.7$ as the full quasar sample. We show the redshift distribution of the full quasar sample in Fig. 1.

\subsection{The methodology}

The non-linear relation between UV and X-ray luminosity of quasars can be parameterized as [111]

$\log L_{\mathrm{X}}=\beta+\gamma \log L_{\mathrm{UV}}$,

where $\log$ denotes $\log _{10} . \beta$ and $\gamma$ are two free parameters determined by the quasar sample. $L_{\mathrm{X}}$ is the monochromatic luminosity at $2 \mathrm{keV}$ and $L_{\mathrm{UV}}$ is the luminosity at $2500 \AA$. The luminosities and fluxes of quasars are related by the luminosity distance, so we rewrite the Eq. (1) as

$$
\begin{aligned}
\log \left(F_{X}\right)= & \beta+(\gamma-1) \log (4 \pi)+\gamma \log \left(F_{U V}\right) \\
& +2(\gamma-1) \log \frac{d_{L}}{\mathrm{Mpc}},
\end{aligned}
$$

where $F_{X}$ and $F_{U V}$ are the X-ray and UV fluxes of quasars, respectively. The luminosity distance $d_{L}$ has the form

$d_{L}=\frac{c(1+z)}{H_{0}} \int_{0}^{z} \frac{d z^{\prime}}{E\left(z^{\prime}\right)}$,

where $z$ is the redshift, $\mathrm{c}$ is the speed of light, and $H_{0}$ is the Hubble constant. The expression of $E(z)$ varies with cosmological models.

In the Finslerian cosmological model, the scale factor $a$ takes the form [112]

$a=\frac{1+A_{D} \cos \theta}{1+z}$,

where $A_{D}$ is the dipole amplitude. $\theta$ is the angle between the preferred direction in the Finslerian cosmological model and the positions of quasars. Correspondingly, $E(z)$ in the Finslerian cosmological model takes the form

$$
E(z)=\sqrt{\Omega_{m 0}(1+z)^{3}\left(1+A_{D} \cos \theta\right)^{-3}+1-\Omega_{m 0}} .
$$


The dipole fitting (DF) method proposed by Mariano and Perivolaropoulos [10] is widely used to test the cosmic anisotropy. In this paper, we consider a dipole modulation to the theoretical distance modulus in the $\Lambda \mathrm{CDM}$ model, namely

$\tilde{\mu}_{t h}=\mu_{t h} \times\left(1+A_{D}(\hat{\mathbf{n}} \cdot \hat{\mathbf{p}})\right)$,

where $A_{D}$ is the dipole amplitude. $\hat{\mathbf{n}}$ and $\hat{\mathbf{p}}$ are the dipole direction and the unit vector pointing to a quasar, respectively. In the galactic coordinate system, the dipole direction $\hat{\mathbf{n}}$ takes the form

$\hat{\boldsymbol{n}}=\cos (b) \cos (l) \hat{\boldsymbol{i}}+\cos (b) \sin (l) \hat{\boldsymbol{j}}+\sin (b) \hat{\boldsymbol{k}}$,

where $\hat{\boldsymbol{i}}, \hat{\boldsymbol{j}}$, and $\hat{\boldsymbol{k}}$ are the unit vectors along the axis in the Cartesian coordinates system. $l$ is the galactic longitude and $b$ is the galactic latitude. The unit vector $\hat{\boldsymbol{p}}_{i}$ pointing to the position of $i$ th quasar can be written as

$\hat{\boldsymbol{p}}_{i}=\cos \left(b_{i}\right) \cos \left(l_{i}\right) \hat{\boldsymbol{i}}+\cos \left(b_{i}\right) \sin \left(l_{i}\right) \hat{\boldsymbol{j}}+\sin \left(b_{i}\right) \hat{\boldsymbol{k}}$.

Considering the expression of distance modulus in the $\Lambda \mathrm{CDM}$ model,

$\mu_{t h}=5 \log \frac{d_{L}}{\mathrm{Mpc}}+25$,

We can write the dipole-modulated distance modulus as

$\tilde{\mu}_{t h}=5 \log \frac{d_{L}}{\mathrm{Mpc}}+25$.

Plugging Eq. (6) into Eq. (10), we obtain

$\tilde{\log } \frac{d_{L}}{\mathrm{Mpc}}=\left(\log \frac{d_{L}}{\mathrm{Mpc}}+5\right) \times\left(1+A_{D}(\hat{\mathbf{n}} \cdot \hat{\mathbf{p}})\right)-5$.

We combine Eqs. (11) and (2) and obtain the theoretical Xray flux in the dipole-modulated $\Lambda \mathrm{CDM}$ model,

$$
\begin{aligned}
\tilde{\log }\left(F_{X}\right)= & \beta+(\gamma-1) \log (4 \pi)+\gamma \log \left(F_{U V}\right) \\
& +2(\gamma-1)\left(\left(\log \frac{d_{L}}{\mathrm{Mpc}}+5\right)\left(1+A_{D}(\hat{\mathbf{n}} \cdot \hat{\mathbf{p}})\right)-5\right) .
\end{aligned}
$$

To explore the whole cosmological parameter space, we employ the likelihood function

$\ln (\mathrm{LF})=-\frac{1}{2} \sum_{i=1}^{N}\left[\frac{\left[\log \left(F_{X, i}^{\mathrm{obs}}\right)-\log \left(F_{X, i}^{\mathrm{th}}\right)\right]^{2}}{s_{i}^{2}}+\ln \left(2 \pi s_{i}^{2}\right)\right]$, where $s_{i}^{2}=\sigma_{i}^{2}+\delta^{2} . \sigma_{i}$ is the error of the observed flux $F_{X, i}^{\mathrm{obs}}$ and $\delta$ is the global intrinsic dispersion. $F_{X, i}^{\mathrm{th}}$ represents the theoretical flux at the redshift $z_{i}$ in the dipole-modulated $\Lambda \mathrm{CDM}$ model or Finslerian cosmological model.

\section{Results}

In this paper, we use the Markov chain Monte Carlo (MCMC) method implemented in emcee ${ }^{1}$ [113] to explore the whole parameters space. Emcee is an Affine Invariant Markov chain Monte Carlo Ensemble sampler, which is widely used to constrain parameters in astrophysics and cosmology. In the dipole-modulated $\Lambda \mathrm{CDM}$ model and Finslerian cosmological model, the free parameters are the matter density $\Omega_{m}$, the dipole amplitude $A_{D}$, the dipole direction $(l, b)$, and the three parameters $\beta, \gamma$, and $\delta$ related to the quasar sample. The flat priors of the free parameters are $\Omega_{m} \sim[0,1], \beta \sim$ $[5,16], \gamma \sim[0.3,0.8], \delta \sim[0.1,0.3], A_{D} \sim[0,0.5], l \sim$ $[0,360], b \sim[-90,90]$.

We use a tomographic method to study the evolution of dipole parameters with redshift. The cutting redshifts are

$z_{\text {cut }}=1.0,1.1,1.2,1.3,1.4,1.5,1.7,1.9,2.1,2.3,2.4,2.5,3.0$.

We divide the quasar sample into two subsets, i.e., $z \leq z_{\text {cut }}$ and $z>z_{\text {cut }}$ by different cutting redshifts $z_{\text {cut }}$. The results of the dipole-modulated $\Lambda \mathrm{CDM}$ model and Finslerian cosmological model are summarized in Tables 1 and 2 .

From Tables 1 and 2, we can see that the results of the two cosmological models are similar except for the dipole amplitude $A_{D}$. The results of parameters $\beta, \gamma$, and $\delta$ from most subsets $z \leq z_{\text {cut }}$ are consistent within statistical uncertainties, respectively. The constraints of matter density $\Omega_{m}$ are very weak from all the subsets. In the Finslerian cosmological model, the results of matter density $\Omega_{m}$ from the subsets $z \leq 1.0,1.1$ are consistent with ones from the Pantheon sample and CMB, but with large uncertainties.

The dipole amplitudes $A_{D}$ of the two cosmological models from the subsets $z \leq z_{c u t}$ are very weak. The upper limit of dipole amplitude $A_{D}$ in the Finslerian cosmological model is about a hundred times larger than that in the $\Lambda \mathrm{CDM}$ model. Though the constraints of matter density $\Omega_{m}$ are very weak from the subsets $z>z_{\text {cut }}$, the results of the dipole amplitude $A_{D}$ show that quasars at a higher redshift range may provide more detailed information about the dipole amplitude $A_{D}$.

Because of the large uncertainties, the results of dipole parameter $l$ from all the subsets $z \leq z_{c u t}$ are consistent. But the central values of parameter $l$ from some subsets have a sudden change. For the dipole-modulated $\Lambda \mathrm{CDM}$

\footnotetext{
${ }^{1} \mathrm{https}: / / \mathrm{emcee} \cdot$ readthedocs.io/en/stable/.
} 
Table 1 The $68 \%$ confidence level constraints of the parameters in the dipole-modulated $\Lambda$ CDM model with different cutting redshifts. If the upper or lower limit of a parameter exists, we show the $95 \%$ confidence level of the parameter

\begin{tabular}{|c|c|c|c|c|c|c|c|c|c|}
\hline Model & Quasar subset & $N$ & $\Omega_{m}$ & $\beta$ & $\gamma$ & $\delta$ & $10^{3} A_{D}$ & $l\left[^{\circ}\right]$ & $b\left[^{\circ}\right]$ \\
\hline \multirow[t]{27}{*}{$\Lambda \mathrm{CDM}$} & $z \leq 1.0$ & 443 & $>0.189$ & $9.037_{-0.714}^{+0.766}$ & $0.573_{-0.027}^{+0.023}$ & $0.235_{-0.008}^{+0.009}$ & $<1.316$ & $120.00_{-101.84}^{+80.17}$ & $-0.91_{-67.53}^{+27.33}$ \\
\hline & $z \leq 1.1$ & 571 & $>0.198$ & $8.521_{-0.708}^{+0.717}$ & $0.591_{-0.025}^{+0.023}$ & $0.243_{-0.008}^{+0.007}$ & $<1.632$ & $112.74_{-74.83}^{+68.24}$ & $-10.01_{-52.58}^{+20.05}$ \\
\hline & $z \leq 1.2$ & 719 & $>0.263$ & $8.715_{-0.617}^{+0.614}$ & $0.583_{-0.020}^{+0.021}$ & $0.237_{-0.006}^{+0.007}$ & $<1.160$ & $112.72_{-99.74}^{+73.46}$ & $-28.20_{-47.30}^{+20.07}$ \\
\hline & $z \leq 1.3$ & 831 & $>0.269$ & $8.882_{-0.494}^{+0.663}$ & $0.577_{-0.022}^{+0.018}$ & $0.239_{-0.006}^{+0.006}$ & $<1.051$ & $130.92_{-85.20}^{+91.67}$ & $-26.37_{-55.09}^{+17.24}$ \\
\hline & $z \leq 1.4$ & 943 & $>0.312$ & $9.021_{-0.554}^{+0.525}$ & $0.573_{-0.018}^{+0.019}$ & $0.236_{-0.006}^{+0.005}$ & $<0.899$ & $138.18_{-78.17}^{+117.89}$ & $-4.55_{-67.62}^{+22.02}$ \\
\hline & $z \leq 1.5$ & 1037 & $>0.339$ & $8.628_{-0.490}^{+0.500}$ & $0.585_{-0.015}^{+0.018}$ & $0.238_{-0.005}^{+0.006}$ & $<0.744$ & $127.28_{-81.31}^{+142.73}$ & $-20.91_{-62.79}^{+30.11}$ \\
\hline & $z \leq 1.7$ & 1270 & $>0.445$ & $8.453_{-0.466}^{+0.426}$ & $0.591_{-0.015}^{+0.015}$ & $0.235_{-0.005}^{+0.005}$ & $<0.661$ & $152.70_{-75.27}^{+137.74}$ & $13.64_{-47.64}^{+51.37}$ \\
\hline & $z \leq 1.9$ & 1446 & $>0.586$ & $8.100_{-0.377}^{+0.420}$ & $0.603_{-0.014}^{+0.012}$ & $0.235_{-0.004}^{+0.005}$ & $<0.623$ & $293.64_{-163.58}^{+65.28}$ & $13.65_{-30.97}^{+65.38}$ \\
\hline & $z \leq 2.1$ & 1593 & $>0.660$ & $8.277_{-0.311}^{+0.430}$ & $0.597_{-0.013}^{+0.012}$ & $0.232_{-0.005}^{+0.004}$ & $<0.622$ & $300.91_{-155.31}^{+66.76}$ & $10.02_{-21.56}^{+70.60}$ \\
\hline & $z \leq 2.3$ & 1712 & $>0.716$ & $8.288_{-0.347}^{+0.331}$ & $0.597_{-0.011}^{+0.011}$ & $0.231_{-0.004}^{+0.004}$ & $<0.593$ & $308.19_{-74.48}^{+154.99}$ & $13.64_{-32.86}^{+61.39}$ \\
\hline & $z \leq 2.4$ & 1776 & $>0.736$ & $8.180_{-0.322}^{+0.333}$ & $0.601_{-0.012}^{+0.010}$ & $0.230_{-0.004}^{+0.004}$ & $<0.567$ & $279.09_{-31.50}^{+209.77}$ & $15.47_{-28.68}^{+63.49}$ \\
\hline & $z \leq 2.5$ & 1812 & $>0.741$ & $8.107_{-0.342}^{+0.324}$ & $0.603_{-0.011}^{+0.011}$ & $0.229_{-0.004}^{+0.004}$ & $<0.544$ & $145.45_{-118.87}^{+119.22}$ & $33.65_{-35.47}^{+51.70}$ \\
\hline & $z \leq 3.0$ & 1943 & $>0.780$ & $7.787_{-0.312}^{+0.297}$ & $0.614_{-0.010}^{+0.010}$ & $0.227_{-0.004}^{+0.004}$ & $<0.615$ & $112.74_{-178.99}^{+60.65}$ & $39.10_{-22.43}^{+50.47}$ \\
\hline & $1.7<z \leq 2.4$ & 506 & $>0.196$ & $10.728_{-0.690}^{+0.602}$ & $0.521_{-0.020}^{+0.022}$ & $0.204_{-0.008}^{+0.007}$ & $<0.861$ & $40.01_{-118.04}^{+100.05}$ & $10.01_{-28.25}^{+64.89}$ \\
\hline & $z>1.0$ & 1595 & $>0.762$ & $7.575_{-0.343}^{+0.360}$ & $0.621_{-0.012}^{+0.011}$ & $0.224_{-0.005}^{+0.004}$ & $<0.752$ & $298.17_{-127.45}^{+79.48}$ & $55.46_{-31.97}^{+34.50}$ \\
\hline & $z>1.1$ & 1467 & $>0.758$ & $7.792_{-0.361}^{+0.368}$ & $0.615_{-0.012}^{+0.012}$ & $0.218_{-0.004}^{+0.005}$ & $0.435_{-0.222}^{+0.264}$ & $296.36_{-103.73}^{+68.85}$ & $68.19_{-32.28}^{+21.79}$ \\
\hline & $z>1.2$ & 1319 & $>0.716$ & $7.913_{-0.368}^{+0.395}$ & $0.610_{-0.013}^{+0.012}$ & $0.218_{-0.005}^{+0.005}$ & $0.558_{-0.255}^{+0.216}$ & $280.01_{-129.05}^{+61.68}$ & $77.42_{-31.44}^{+12.58}$ \\
\hline & $z>1.3$ & 1207 & $>0.691$ & $8.116_{-0.415}^{+0.384}$ & $0.604_{-0.013}^{+0.014}$ & $0.213_{-0.005}^{+0.005}$ & $0.537_{-0.234}^{+0.251}$ & $305.46_{-111.48}^{+79.54}$ & $77.41_{-33.81}^{+12.58}$ \\
\hline & $z>1.4$ & 1095 & $>0.647$ & $8.198_{-0.366}^{+0.455}$ & $0.602_{-0.015}^{+0.012}$ & $0.211_{-0.005}^{+0.005}$ & $0.507_{-0.253}^{+0.254}$ & $305.45_{-100.98}^{+108.08}$ & $75.46_{-32.40}^{+14.43}$ \\
\hline & $z>1.5$ & 1001 & $>0.629$ & $8.647_{-0.416}^{+0.406}$ & $0.588_{-0.014}^{+0.013}$ & $0.206_{-0.005}^{+0.005}$ & $0.534_{-0.256}^{+0.232}$ & $290.90_{-169.69}^{+46.23}$ & $75.57_{-27.62}^{+14.39}$ \\
\hline & $z>1.7$ & 768 & $>0.540$ & $9.275_{-0.452}^{+0.448}$ & $0.568_{-0.014}^{+0.015}$ & $0.198_{-0.006}^{+0.006}$ & $0.397_{-0.268}^{+0.290}$ & $350.90_{-72.48}^{+128.57}$ & $66.40_{-36.35}^{+22.68}$ \\
\hline & $z>1.9$ & 592 & $>0.516$ & $9.303_{-0.456}^{+0.535}$ & $0.567_{-0.017}^{+0.015}$ & $0.187_{-0.007}^{+0.007}$ & $0.450_{-0.340}^{+0.267}$ & $16.37_{-83.65}^{+114.17}$ & $64.55_{-33.39}^{+25.40}$ \\
\hline & $z>2.1$ & 445 & $>0.365$ & $9.329_{-0.555}^{+0.547}$ & $0.567_{-0.018}^{+0.018}$ & $0.181_{-0.007}^{+0.009}$ & $0.677_{-0.373}^{+0.433}$ & $89.10_{-96.27}^{+64.21}$ & $48.19_{-9.68}^{+39.05}$ \\
\hline & $z>2.3$ & 326 & $>0.258$ & $8.706_{-0.676}^{+0.647}$ & $0.589_{-0.021}^{+0.022}$ & $0.169_{-0.008}^{+0.011}$ & $1.121_{-0.442}^{+0.576}$ & $130.91_{-79.52}^{+72.22}$ & $57.63_{-11.83}^{+25.69}$ \\
\hline & $z>2.4$ & 262 & $>0.259$ & $9.241_{-0.683}^{+0.786}$ & $0.573_{-0.024}^{+0.023}$ & $0.170_{-0.009}^{+0.012}$ & $1.061_{-0.404}^{+0.465}$ & $329.09_{-50.69}^{+174.40}$ & $79.11_{-25.72}^{+10.89}$ \\
\hline & $z>2.5$ & 226 & $>0.219$ & $9.701_{-0.852}^{+0.698}$ & $0.560_{-0.025}^{+0.024}$ & $0.173_{-0.012}^{+0.011}$ & $1.061_{-0.404}^{+0.465}$ & $309.09_{-62.86}^{+170.21}$ & $77.30_{-24.28}^{+12.63}$ \\
\hline & $z>3.0$ & 95 & $>0.152$ & $10.568_{-1.630}^{+1.605}$ & $0.532_{-0.051}^{+0.052}$ & $0.203_{-0.019}^{+0.020}$ & $1.096_{-0.471}^{+0.456}$ & $309.10_{-96.97}^{+103.05}$ & $19.09_{-34.67}^{+60.52}$ \\
\hline
\end{tabular}

model, the central values of parameter $l$ from the subsets $z \leq 1.9,2.1,2.3,2.4$ and $z>1.9,2.1,2.3$ have a sudden change. For the Finslerian cosmological model, the central values of parameter $l$ from the subsets $z \leq 1.9,2.1,2.3$ and $z>1.9,2.1,2.3$ have a sudden change.

We analyze the subset $1.7<z \leq 2.4$ and show the results in Tables 1 and 2. The central value of parameter $l$ from subsets $1.7<z \leq 2.4$ is $40.01^{\circ}$ in the dipolemodulated $\Lambda \mathrm{CDM}$ model and $83.63^{\circ}$ in the Finslerian cosmological model. In the dipole-modulated $\Lambda$ CDM model, the results of parameters $\beta, \gamma$, and $\delta$ are $\beta=10.728_{-0.690}^{+0.602}$, $\gamma=0.521_{-0.020}^{+0.022}$, and $\delta=0.204_{-0.008}^{+0.007}$. In the Finslerian cosmological model, the results of parameters $\beta, \gamma$, and $\delta$ are $\beta=10.615_{-0.604}^{+0.706}, \gamma=0.526_{-0.024}^{+0.018}$, and $\delta=0.203_{-0.006}^{+0.009}$. For parameters $\beta, \gamma$, and $\delta$, the differences between the results from subsets $1.7<z \leq 2.4$ and the ones from the subsets $z \leq 1.90,2.10,2.30,2.40$ and $z>1.90,2.10,2.30$ are more than $1 \sigma$ level. The central value of parameter $l$ has a sudden change in some subsets may be due to the big deviations of parameters $\beta, \gamma$, and $\delta$.

The dipole directions derived from two subsets divided by different cutting redshifts are consistent within statistical uncertainties except for the subsets $z \leq 1.1$ and $z>1.1$. For brevity, the subset $z \leq 1.1$ is shortened to Q1 and the subset $z>1.1$ is shortened to Q2. In Fig. 2, We show the distributions of subsets Q1 and Q2 in the galactic coordinate system. In the dipolemodulated $\Lambda \mathrm{CDM}$ model, the dipole direction from the subset Q1 is $(l, b)=\left(112.74_{-74.83^{\circ}}^{\circ},-10.01_{-52.58^{\circ}}^{\circ}\right)$ and the dipole direction from the subset $\mathrm{Q} 2$ is $(l, b)=$ $\left(296.36_{-103.73^{\circ}}^{\circ+68.85^{\circ}}, 68.19_{-32.28^{\circ}}^{\circ}\right)$. In the Finslerian cosmo- 
Table 2 The $68 \%$ confidence level constraints of the parameters in the Finslerian cosmological model with different cutting redshifts. If the upper or lower limit of a parameter exists, we show the $95 \%$ confidence level of the parameter

\begin{tabular}{|c|c|c|c|c|c|c|c|c|c|}
\hline Model & Quasar subset & $N$ & $\Omega_{m}$ & $\beta$ & $\gamma$ & $\delta$ & $A_{D}$ & $l\left[^{\circ}\right]$ & $b\left[^{\circ}\right]$ \\
\hline \multirow[t]{27}{*}{ Finslerian } & $z \leq 1.0$ & 443 & $0.505_{-0.257}^{+0.347}$ & $9.066_{-0.753}^{+0.751}$ & $0.572_{-0.025}^{+0.026}$ & $0.234_{-0.007}^{+0.008}$ & $<0.230$ & $116.37_{-94.95}^{+80.08}$ & $-2.73_{-63.21}^{+27.27}$ \\
\hline & $z \leq 1.1$ & 571 & $0.596_{-0.376}^{+0.218}$ & $8.473_{-0.700}^{+0.710}$ & $0.593_{-0.025}^{+0.022}$ & $0.242_{-0.007}^{+0.008}$ & $<0.273$ & $109.09_{-66.59}^{+68.75}$ & $-13.64_{-49.61}^{+20.58}$ \\
\hline & $z \leq 1.2$ & 719 & $0.648_{-0.266}^{+0.276}$ & $8.741_{-0.654}^{+0.596}$ & $0.583_{-0.020}^{+0.022}$ & $0.236_{-0.006}^{+0.007}$ & $<0.163$ & $109.07_{-98.57}^{+79.76}$ & $-51.83_{-29.27}^{+38.00}$ \\
\hline & $z \leq 1.3$ & 831 & $0.566_{-0.107}^{+0.408}$ & $8.945_{-0.595}^{+0.592}$ & $0.575_{-0.020}^{+0.020}$ & $0.239_{-0.006}^{+0.006}$ & $<0.148$ & $127.29_{-86.48}^{+86.59}$ & $-20.92_{-54.70}^{+16.83}$ \\
\hline & $z \leq 1.4$ & 943 & $>0.264$ & $9.017_{-0.506}^{+0.554}$ & $0.573_{-0.017}^{+0.019}$ & $0.236_{-0.005}^{+0.006}$ & $<0.113$ & $138.18_{-83.28}^{+110.78}$ & $-6.36_{-60.95}^{+24.16}$ \\
\hline & $z \leq 1.5$ & 1037 & $>0.287$ & $8.665_{-0.557}^{+0.443}$ & $0.586_{-0.016}^{+0.018}$ & $0.238_{-0.005}^{+0.006}$ & $<0.086$ & $130.91_{-110.89}^{+115.84}$ & $-17.27_{-57.85}^{+36.14}$ \\
\hline & $z \leq 1.7$ & 1270 & $>0.402$ & $8.409_{-0.424}^{+0.449}$ & $0.594_{-0.016}^{+0.014}$ & $0.235_{-0.005}^{+0.005}$ & $<0.074$ & $149.09_{-76.65}^{+139.04}$ & $11.82_{-43.42}^{+51.48}$ \\
\hline & $z \leq 1.9$ & 1446 & $>0.564$ & $8.111_{-0.420}^{+0.375}$ & $0.604_{-0.013}^{+0.013}$ & $0.236_{-0.005}^{+0.004}$ & $<0.062$ & $275.46_{-144.50}^{+87.04}$ & $13.64_{-34.10}^{+61.47}$ \\
\hline & $z \leq 2.1$ & 1593 & $>0.629$ & $8.285_{-0.363}^{+0.358}$ & $0.597_{-0.012}^{+0.012}$ & $0.233_{-0.004}^{+0.004}$ & $<0.061$ & $297.27_{-144.37}^{+72.71}$ & $15.45_{-30.36}^{+61.70}$ \\
\hline & $z \leq 2.3$ & 1712 & $>0.706$ & $8.317_{-0.366}^{+0.317}$ & $0.597_{-0.011}^{+0.012}$ & $0.232_{-0.004}^{+0.004}$ & $<0.053$ & $297.27_{-104.95}^{+123.55}$ & $15.46_{-30.10}^{+62.73}$ \\
\hline & $z \leq 2.4$ & 1776 & $>0.725$ & $8.171_{-0.318}^{+0.350}$ & $0.601_{-0.012}^{+0.010}$ & $0.230_{-0.004}^{+0.004}$ & $<0.052$ & $100.91_{-217.06}^{+28.75}$ & $22.73_{-33.06}^{+58.39}$ \\
\hline & $z \leq 2.5$ & 1812 & $>0.729$ & $8.138_{-0.366}^{+0.289}$ & $0.602_{-0.009}^{+0.012}$ & $0.230_{-0.004}^{+0.004}$ & $<0.054$ & $116.36_{-191.76}^{+47.79}$ & $19.10_{-27.16}^{+62.70}$ \\
\hline & $z \leq 3.0$ & 1943 & $>0.781$ & $7.818_{-0.341}^{+0.266}$ & $0.613_{-0.009}^{+0.011}$ & $0.227_{-0.004}^{+0.004}$ & $<0.057$ & $130.91_{-188.28}^{+50.25}$ & $37.29_{-24.21}^{+49.54}$ \\
\hline & $1.7<z \leq 2.4$ & 506 & $>0.119$ & $10.615_{-0.604}^{+0.706}$ & $0.526_{-0.024}^{+0.018}$ & $0.203_{-0.006}^{+0.009}$ & $<0.102$ & $83.63_{-141.55}^{+68.07}$ & $11.82_{-44.72}^{+51.99}$ \\
\hline & $z>1.0$ & 1595 & $>0.745$ & $7.605_{-0.369}^{+0.344}$ & $0.620_{-0.011}^{+0.012}$ & $0.224_{-0.005}^{+0.004}$ & $<0.069$ & $294.54_{-107.58}^{+109.15}$ & $62.73_{-41.31}^{+25.02}$ \\
\hline & $z>1.1$ & 1467 & $>0.751$ & $7.794_{-0.354}^{+0.369}$ & $0.614_{-0.012}^{+0.012}$ & $0.219_{-0.005}^{+0.004}$ & $0.040_{-0.020}^{+0.024}$ & $296.36_{-103.80}^{+79.05}$ & $70.00_{-32.58}^{+19.94}$ \\
\hline & $z>1.2$ & 1319 & $>0.722$ & $7.978_{-0.424}^{+0.318}$ & $0.609_{-0.010}^{+0.014}$ & $0.218_{-0.004}^{+0.005}$ & $0.050_{-0.022}^{+0.024}$ & $280.00_{-130.81}^{+63.19}$ & $71.85_{-26.66}^{+18.07}$ \\
\hline & $z>1.3$ & 1207 & $>0.681$ & $8.124_{-0.425}^{+0.366}$ & $0.604_{-0.012}^{+0.014}$ & $0.214_{-0.005}^{+0.005}$ & $0.051_{-0.023}^{+0.022}$ & $305.45_{-97.87}^{+91.33}$ & $77.51_{-33.54}^{+12.48}$ \\
\hline & $z>1.4$ & 1095 & $>0.623$ & $8.199_{-0.390}^{+0.428}$ & $0.602_{-0.014}^{+0.013}$ & $0.211_{-0.004}^{+0.006}$ & $0.047_{-0.024}^{+0.024}$ & $305.46_{-137.00}^{+73.35}$ & $75.47_{-32.05}^{+14.53}$ \\
\hline & $z>1.5$ & 1001 & $>0.637$ & $8.632_{-0.416}^{+0.407}$ & $0.588_{-0.013}^{+0.014}$ & $0.207_{-0.006}^{+0.005}$ & $0.052_{-0.026}^{+0.023}$ & $294.55_{-172.14}^{+43.67}$ & $73.66_{-27.65}^{+16.28}$ \\
\hline & $z>1.7$ & 768 & $>0.515$ & $9.264_{-0.501}^{+0.424}$ & $0.568_{-0.014}^{+0.017}$ & $0.199_{-0.007}^{+0.006}$ & $0.036_{-0.027}^{+0.025}$ & $358.18_{-82.80}^{+117.00}$ & $50.00_{-20.77}^{+39.06}$ \\
\hline & $z>1.9$ & 592 & $>0.499$ & $9.320_{-0.507}^{+0.487}$ & $0.566_{-0.015}^{+0.017}$ & $0.188_{-0.007}^{+0.007}$ & $<0.102$ & $20.00_{-90.66}^{+112.70}$ & $64.55_{-32.58}^{+25.23}$ \\
\hline & $z>2.1$ & 445 & $>0.317$ & $9.280_{-0.579}^{+0.546}$ & $0.569_{-0.017}^{+0.019}$ & $0.181_{-0.008}^{+0.008}$ & $0.067_{-0.040}^{+0.048}$ & $92.73_{-99.19}^{+56.14}$ & $46.41_{-17.88}^{+30.81}$ \\
\hline & $z>2.3$ & 326 & $>0.278$ & $8.653_{-0.640}^{+0.685}$ & $0.590_{-0.021}^{+0.022}$ & $0.171_{-0.009}^{+0.009}$ & $0.113_{-0.050}^{+0.060}$ & $127.30_{-75.08}^{+83.42}$ & $59.09_{-13.21}^{+25.22}$ \\
\hline & $z>2.4$ & 262 & $>0.250$ & $9.226_{-0.764}^{+0.688}$ & $0.573_{-0.022}^{+0.025}$ & $0.172_{-0.011}^{+0.011}$ & $0.109_{-0.046}^{+0.048}$ & $321.81_{-28.95}^{+201.46}$ & $77.32_{-24.11}^{+12.68}$ \\
\hline & $z>2.5$ & 226 & $>0.230$ & $9.547_{-0.757}^{+0.846}$ & $0.561_{-0.026}^{+0.025}$ & $0.172_{-0.012}^{+0.011}$ & $0.109_{-0.048}^{+0.054}$ & $319.99_{-93.81}^{+138.43}$ & $75.51_{-23.92}^{+14.45}$ \\
\hline & $z>3.0$ & 95 & $>0.128$ & $10.504_{-1.678}^{+1.540}$ & $0.530_{-0.047}^{+0.056}$ & $0.204_{-0.019}^{+0.021}$ & $<0.237$ & $305.45_{-119.73}^{+100.78}$ & $17.26_{-27.84}^{+66.24}$ \\
\hline
\end{tabular}

logical model, the dipole direction from the subset Q1 is $(l, b)=\left(109.09_{-66.59^{\circ}}^{\circ},-13.64_{-49.61^{\circ}}^{\circ}\right)$ and the dipole direction from the subset Q2 is $(l, b)=\left(296.36_{-103.80^{\circ}}^{\circ}\right.$, $\left.70.00_{-32.58^{\circ}}^{\circ}\right)$. In each cosmological model, the difference between the two directions from subsets Q1 and Q2 is about $1 \sigma$ level. We summarize the results from the subsets Q1 and Q2 in Table 3 for clarity. We show the marginalized posterior distribution for each cosmological model in Figs. 3 and 4.

From Table 3, we can see that the results of the two cosmological models are similar except for the parameter $A_{D}$. The constraints of matter density $\Omega_{m}$ in the two cosmological models are very weak. For the parameters $\beta$ and $\gamma$ in each cosmological model, the results from subsets Q1 and Q2 are consistent within $1 \sigma$ uncertainties, respectively. For the parameter $\delta$ in each cosmological model, the differences between the results from subset $\mathrm{Q} 1$ and $\mathrm{Q} 2$ are about $2 \sigma$ level.

In the dipole-modulated $\triangle \mathrm{CDM}$ model, the dipole amplitude $A_{D}$ is constrained as $A_{D}<1.632 \times 10^{-3}$ at $95 \% \mathrm{CL}$ with subset Q1, and the dipole amplitude $A_{D}$ is $0.435_{-0.222}^{+0.264} \times$ $10^{-3}$ with subset Q2. In the Finslerian cosmological model, the dipole amplitude $A_{D}$ is constrained as $A_{D}<0.273$ at 95\% CL with subsets $\mathrm{Q} 1$, and the dipole amplitude $A_{D}$ is $0.040_{-0.020}^{+0.024}$ with subsets $\mathrm{Q} 2$. The dipole amplitude $A_{D}$ in the Finslerian cosmological model is about a hundred times larger than that in the $\Lambda \mathrm{CDM}$ model.

The Pantheon sample is combined with the two subsets Q1 and Q2 to test the cosmological models. In Fig. 2, We show the distributions of Pantheon sample in the galactic coordinate system. For brevity, the dataset Q1 + Pantheon is shortened to Q1-P and the dataset Q2 + Pantheon is short- 


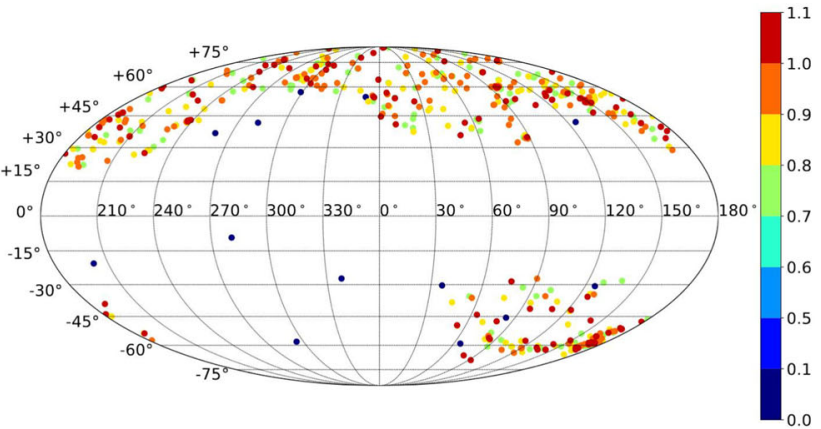

(a) Q1 (Quasar $\mathrm{z} \leq 1.1$ )

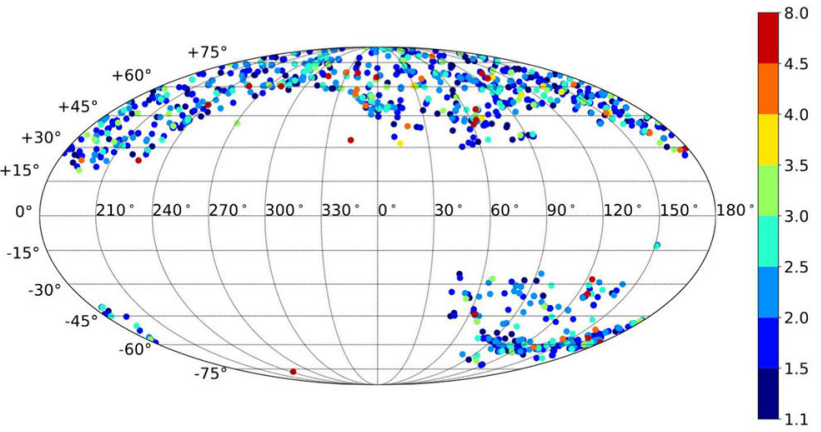

(b) Q2 (Quasar z ¿ 1.1)

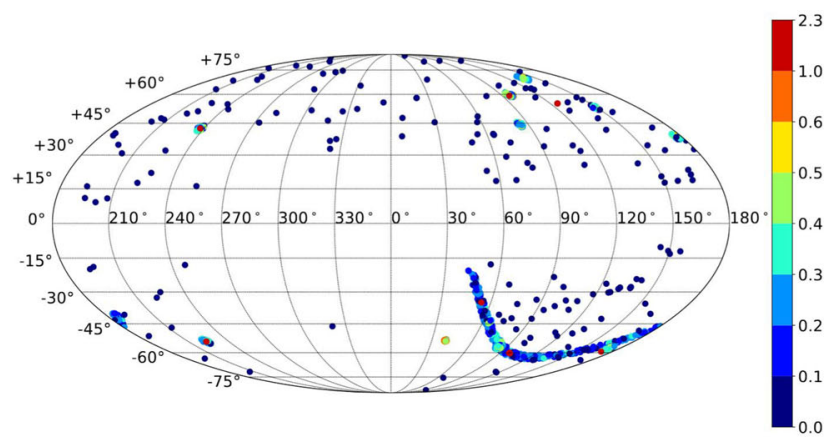

(c) Pantheon

Fig. 2 The distribution of subsets Q1, Q2, and Pantheon sample in the galactic coordinate system. The pseudo-colors represent the redshift of quasars or SNe Ia

ened to Q2-P. The results from the combined datasets are shown in Figs. 3 and 4 and summarized in Table 3. In Figs. 3 and 4, we show the marginalized posterior distribution for each cosmological model. In Table 3, we show the $68 \%$ CL constraints on the parameters in each cosmological model. If the upper or lower limit of a parameter exists, we show the 95\% CL limit of the parameter.

In the dipole-modulated $\Lambda \mathrm{CDM}$ model, the dataset Q1-P gives a dipole direction points to $(l, b)=\left(121.82^{\circ}+66.19^{\circ}\right.$, $\left.-73.64_{-16.36^{\circ}}^{\circ}\right)$. The dipole direction is consistent with the one from the subset Q1 within statistical uncertainties, but the central values of parameter $b$ change a lot. The dipole amplitude is constrained as $A_{D}<0.832 \times 10^{-3}$ at $95 \%$ CL. The results of parameters $\beta, \gamma$, and $\delta$ are $\beta=8.378_{-0.674}^{+0.683}$, $\gamma=0.596_{-0.023}^{+0.022}$, and $\delta=0.242_{-0.007}^{+0.008}$. The matter density is $\Omega_{m}=0.297_{-0.020}^{+0.023}$, which is deviated from the result in the subset $z \leq 1.10$ by $1 \sigma$ level. It is worth noting that the dipole direction in the dataset Q1-P is far away from the one $\left((l, b)=\left(306.00_{-125.98^{\circ}}^{\circ},-23.41_{-54.71^{\circ}}^{\circ}\right)\right)$ in the Pantheon sample [106].

For the dataset Q2-P, the dipole direction is $(l, b)=$ $\left(305.47_{-78.58^{\circ}}^{\circ}, 11.82_{-17.39^{\circ}}^{\circ}\right)$, which is consistent with the one from the subset $\mathrm{Q} 2$. The central value of parameters $b$ in the two dipole directions also change a lot. The dipole amplitude is constrained as $A_{D}<0.818 \times 10^{-3}$ at $95 \%$ CL.
The results of parameters $\beta, \gamma$, and $\delta$ are $\beta=7.545_{-0.382}^{+0.330}$, $\gamma=0.626_{-0.011}^{+0.012}$, and $\delta=0.221_{-0.005}^{+0.004}$. The result of parameter $\delta$ in the dataset Q2-P deviates from that in the dataset $\mathrm{Q} 1-\mathrm{P}$ by $1 \sigma$ level. The matter density is $\Omega_{m}=0.319_{-0.022}^{+0.024}$, which is against the result in the subset Q2.

In the Finslerian cosmological model, the dataset Q1-P gives a dipole direction points to $(l, b)=\left(301.81^{\circ}{ }_{-152.99^{\circ}}\right.$, $\left.-39.09^{\circ}{ }_{-50.73^{\circ}}\right)$. The dipole direction is consistent with the one from the subset Q1 within statistical uncertainties, but the central values of parameter $l$ change a lot. The dipole amplitude is constrained as $A_{D}<0.018$ at $95 \% \mathrm{CL}$. The results of parameters $\beta, \gamma$, and $\delta$ are $\beta=8.319_{-0.698}^{+0.654}, \gamma=$ $0.598_{-0.022}^{+0.023}$, and $\delta=0.243_{-0.008}^{+0.007}$ and the matter density is $\Omega_{m}=0.297_{-0.020}^{+0.023}$.

For the dataset Q2-P, the dipole direction points to $(l, b)=$ $\left(309.09^{\circ}+72.06^{\circ},-19.07^{\circ},-22.96^{\circ}\right)$, which has $1 \sigma$ level deviation from the one in the subset Q2. The dipole amplitude is constrained as $A_{D}<0.019$ at $95 \% \mathrm{CL}$. The results of parameters $\beta, \gamma$, and $\delta$ are $\beta=7.605_{-0.397}^{+0.315}, \gamma=0.624_{-0.011}^{+0.012}$, and $\delta=0.221_{-0.005}^{+0.004}$. The result of parameter $\delta$ deviates from the one in the dataset Q1-P by $1 \sigma$ level. The matter density is $\Omega_{m}=0.319_{-0.022}^{+0.024}$, which is against the result in the subset $\mathrm{Q} 2$.

It is necessary to check whether the inhomogeneous distributions of quasars and SNe Ia have some influence on the 
Table 3 The $68 \%$ confidence level constraints on the parameters in the dipole-modulated $\Lambda$ CDM model and Finslerian cosmological model. The datasets are Q1, Q2, Q1-P, and Q2-P. If the upper or lower limit of a parameter exists, we show the 95\% confidence level of the parameter

\begin{tabular}{|c|c|c|c|c|c|c|c|c|}
\hline Model & Dataset & $\Omega_{m}$ & $\beta$ & $\gamma$ & $\delta$ & $10^{3} A_{D}$ & $l\left[{ }^{\circ}\right]$ & $b\left[^{\circ}\right]$ \\
\hline \multirow[t]{4}{*}{$\Lambda \mathrm{CDM}$} & Q1 & $>0.198$ & $8.521_{-0.708}^{+0.717}$ & $0.591_{-0.025}^{+0.023}$ & $0.243_{-0.008}^{+0.007}$ & $<1.632$ & $112.74_{-74.83}^{+68.24}$ & $-10.01_{-52.58}^{+20.05}$ \\
\hline & Q2 & $>0.758$ & $7.792_{-0.361}^{+0.368}$ & $0.615_{-0.012}^{+0.012}$ & $0.218_{-0.004}^{+0.005}$ & $0.435_{-0.222}^{+0.264}$ & $296.36_{-103.73}^{+68.85}$ & $68.19_{-32.28}^{+21.79}$ \\
\hline & Q1-P & $0.297_{-0.020}^{+0.023}$ & $8.378_{-0.674}^{+0.683}$ & $0.596_{-0.023}^{+0.022}$ & $0.242_{-0.007}^{+0.008}$ & $<0.832$ & $121.82_{-148.87}^{+66.19}$ & $-73.64_{-16.36}^{+40.15}$ \\
\hline & Q2-P & $0.322_{-0.022}^{+0.021}$ & $7.545_{-0.382}^{+0.330}$ & $0.626_{-0.011}^{+0.012}$ & $0.221_{-0.005}^{+0.004}$ & $<0.818$ & $305.47_{-78.58}^{+72.54}$ & $11.82_{-17.39}^{+56.80}$ \\
\hline Model & Dataset & $\Omega_{m}$ & $\beta$ & $\gamma$ & $\delta$ & $A_{D}$ & $l\left[{ }^{\circ}\right]$ & $b\left[^{\circ}\right]$ \\
\hline \multirow[t]{4}{*}{ Finslerian } & Q1 & $0.596_{-0.376}^{+0.218}$ & $8.473_{-0.700}^{+0.710}$ & $0.593_{-0.025}^{+0.022}$ & $0.242_{-0.007}^{+0.008}$ & $<0.273$ & $109.09_{-66.59}^{+68.75}$ & $-13.64_{-49.61}^{+20.58}$ \\
\hline & Q2 & $>0.751$ & $7.794_{-0.354}^{+0.369}$ & $0.614_{-0.012}^{+0.012}$ & $0.219_{-0.005}^{+0.004}$ & $0.040_{-0.020}^{+0.024}$ & $296.36_{-103.80}^{+79.05}$ & $70.00_{-32.58}^{+19.94}$ \\
\hline & Q1-P & $0.297_{-0.020}^{+0.023}$ & $8.319_{-0.698}^{+0.654}$ & $0.598_{-0.022}^{+0.023}$ & $0.243_{-0.008}^{+0.007}$ & $<0.018$ & $301.81_{-152.99}^{+56.80}$ & $-39.09_{-50.73}^{+17.47}$ \\
\hline & Q2-P & $0.319_{-0.022}^{+0.024}$ & $7.605_{-0.397}^{+0.315}$ & $0.624_{-0.011}^{+0.012}$ & $0.221_{-0.005}^{+0.004}$ & $<0.019$ & $309.09_{-119.77}^{+72.06}$ & $-19.09_{-57.63}^{+22.96}$ \\
\hline
\end{tabular}

Fig. 3 The marginalized posterior distribution of the parameters in the dipole-modulated $\Lambda \mathrm{CDM}$ model. The blue lines denote the results from dataset Q1, the orange lines denote the results from dataset Q2, the red lines represent the results from dataset Q1-P, and the green lines represent the results from dataset Q2-P

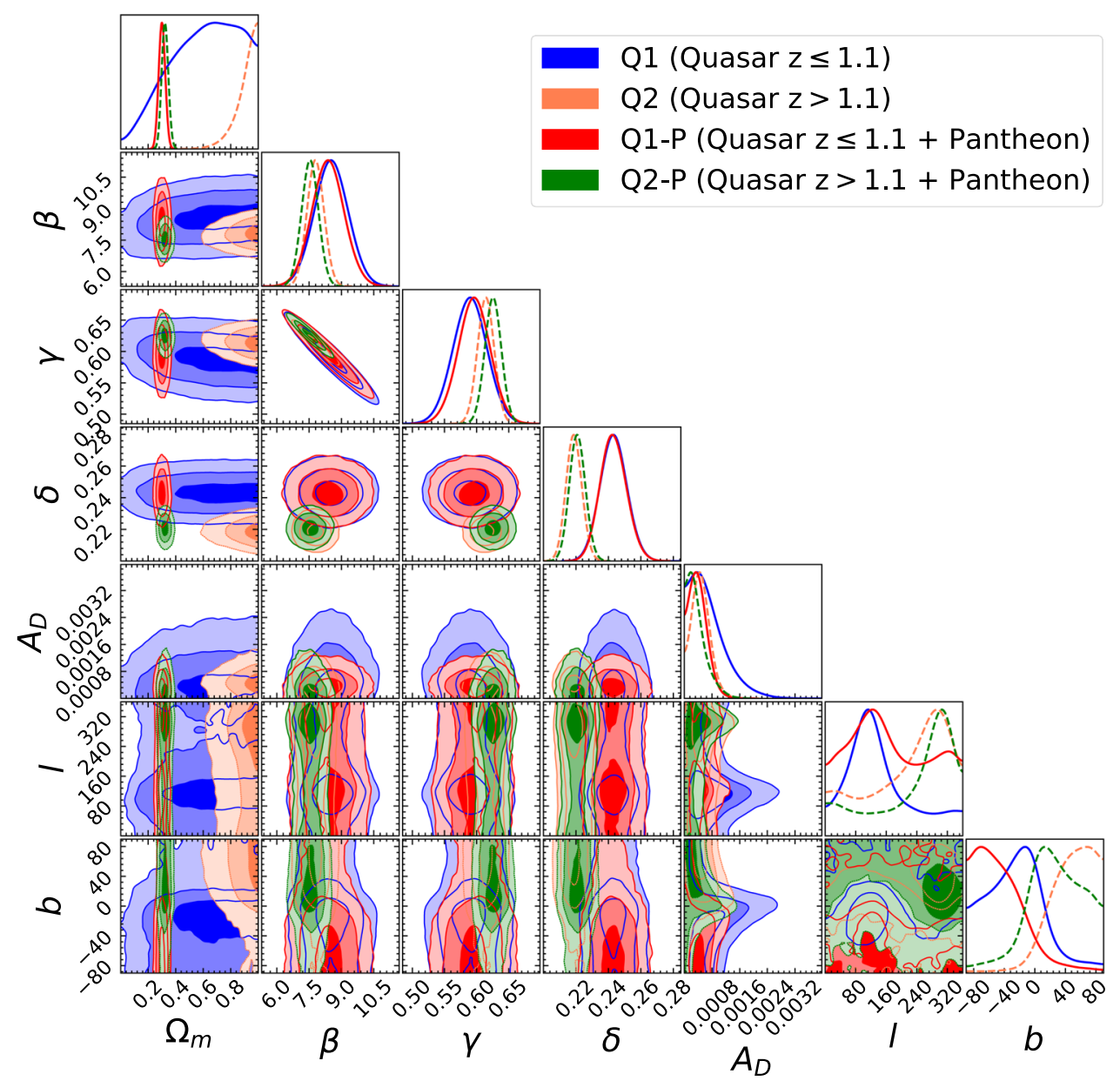

$A_{D}$, we are interested in the dipole directions from the simulations. The results of dipole directions from the simulations are shown in Fig. 5. We can see that the dipole directions derived from the simulations are randomly distributed in the sky. It may imply that the distribution of quasars and SNe Ia have significant effects on the dipole direction derived from the dataset Q1-P. We also construct 200 simulations based on dipole amplitude $A_{D}$ and only can give the upper limit of 
Fig. 4 The marginalized posterior distribution of the parameters in the Finslerian cosmological model. The blue lines denote the results from dataset Q1, the orange lines denote the results from dataset Q2, the red lines represent the results from dataset Q1-P, and the green lines represent the results from dataset $\mathrm{Q} 2-\mathrm{P}$

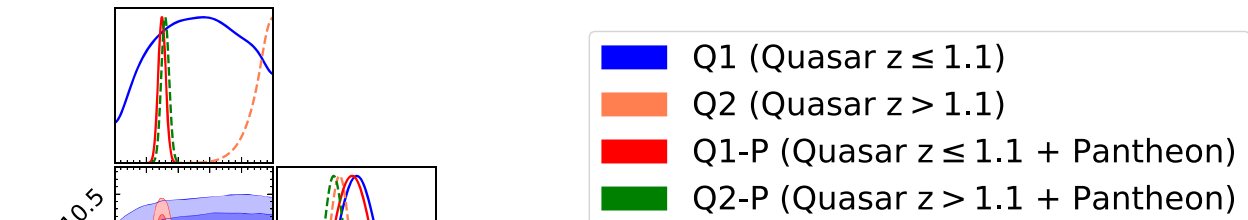

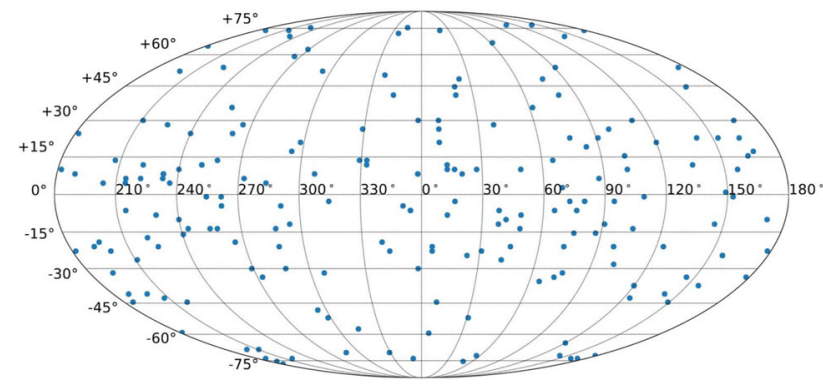

Fig. 5 The distributions of dipole directions derived from the 200 simulations in the galactic coordinate system

the dataset Q2-P and the combination of quasar and Pantheon samples, respectively. The results are similar to ones of the simulations based on the dataset Q1-P.

In the end, we make some comparisons between the dipole directions shown in Table 3 with preferred directions derived from other samples. The preferred directions derived from different samples are summarized in Table 4 and shown in Fig. 6. Most of the preferred directions derived from different samples are located in a relatively small part of the south galactic hemisphere and they are consistent within sta- tistical uncertainties. The dipole direction derived from the dataset Q2-P in the dipole-modulated $\Lambda$ CDM model and the dipole directions derived from the datasets Q1-P and Q2-P in the Finslerian cosmological model are consistent with most preferred directions within statistical uncertainties. The dipole directions derived from the dataset Q2 in the dipole-modulated $\Lambda \mathrm{CDM}$ model and Finslerian cosmological model are close to each other. The two directions are also close to the preferred directions derived from CMB octopole, quasar alignment, and the combination of Pantheon sample and 1421 quasars. The dipole directions derived from the dataset $\mathrm{Q} 1$ in the dipole-modulated $\Lambda \mathrm{CDM}$ model and Finslerian cosmological model are also close to each other. There are only two preferred directions around them. One is the preferred direction derived from the Pantheon sample with the $\mathrm{HC}$ method and the other is the preferred direction derived from the combination of Union2 and 67 GRBs with the DF method. The dipole direction derived from the dataset Q1-P in the dipole-modulated $\Lambda \mathrm{CDM}$ model is far away from the one derived from the dataset Q1-P in the Finslerian cosmological model. This may be due to the difference between the two cosmological models. 
Table 4 The preferred directions derived from different samples

\begin{tabular}{|c|c|c|c|}
\hline Model & Data & Direction $\left(l\left[^{\circ}\right], b\left[^{\circ}\right]\right)$ & References \\
\hline \multirow[t]{22}{*}{$\Lambda \mathrm{CDM}$} & Q1 (DF) & $112.74_{-74.83}^{+68.24},-10.01_{-52.58}^{+20.05}$ & This paper \\
\hline & Q2 (DF) & $296.36_{-103.73}^{+68.85}, 68.19_{-32.28}^{+21.79}$ & This paper \\
\hline & Q1 + Pantheon (DF) & $121.82_{-148.87}^{+66.19},-73.64_{-16.36}^{+40.15}$ & This paper \\
\hline & $\mathrm{Q} 2$ + Pantheon (DF) & $305.47_{-78.58}^{+72.54}, 11.82_{-17.39}^{+56.80}$ & This paper \\
\hline & Union2 (HC) & $309.4 \pm 18.0,-15.1 \pm 11.5$ & {$[92]$} \\
\hline & Union2 (DF) & $309.0 \pm 22.4,-19.3 \pm 12.9$ & {$[10]$} \\
\hline & Union2.1 (HC) & $241.9,-19.5$ & [17] \\
\hline & Union2.1 (DF) & $307.1 \pm 16.2,-14.3 \pm 10.1$ & {$[96]$} \\
\hline & JLA (HC) $\max$ & $23.49_{-12.83}^{+21.62},-2.25_{-22.68}^{+3.80}$ & {$[114]$} \\
\hline & JLA (HC) submax & $299.47_{-23.39}^{+46.13},-28.39_{-17.01}^{+6.52}$ & [114] \\
\hline & JLA (DF) & $316_{-100}^{+107},-5_{-60}^{+41}$ & {$[101]$} \\
\hline & Pantheon (HC) & $123.05_{-4.22}^{+11.25}, 4.78_{-8.36}^{+1.80}$ & [107] \\
\hline & Pantheon (DF) & $306.00_{-125.01}^{+82.95},-34.20_{-54.93}^{+16.82}$ & {$[107]$} \\
\hline & Pantheon + 1421Quasars (HC) & $316.08_{-129.48}^{+27.41}, 4.53_{-64.06}^{+26.29}$ & {$[87]$} \\
\hline & Pantheon + 1421Quasars (DF) & $327.55 \pm 32.45,51.01 \pm 26.50$ & [87] \\
\hline & 67 GRBs (DF) & $336_{-233}^{+33},-5_{-26}^{+34}$ & {$[13]$} \\
\hline & Union2 + 67 GRBs (DF) & $129_{-23}^{+16}, 16_{-10}^{+17}$ & [13] \\
\hline & Union2.1 + 116 GRBs (DF) & $309.2 \pm 15.8,-8.6 \pm 10.5$ & [108] \\
\hline & Keck + VLT (DF) & $320.5 \pm 11.8,-11.7 \pm 7.5$ & {$[10]$} \\
\hline & Galaxy rotation curves (HC) & $175.5_{-10}^{+6},-6.5_{-3}^{+8}$ & {$[18]$} \\
\hline & Galaxy rotation curves (DF) & $171.30 \pm 7.18,-15.41 \pm 4.87$ & [19] \\
\hline & Galaxy cluster & $303,-27$ & [53] \\
\hline \multirow[t]{6}{*}{-} & CMB dipole & $263.99 \pm 0.14,48.26 \pm 0.03$ & [115] \\
\hline & CMB quadrupole & 240,63 & {$[24,116]$} \\
\hline & CMB octopole & 308,63 & {$[24]$} \\
\hline & $\Delta \alpha / \alpha$ & $330,-13$ & {$[8]$} \\
\hline & Quasar alignment & 267,69 & {$[21,117]$} \\
\hline & Velocity flows & 282,6 & {$[118,119]$} \\
\hline \multirow[t]{6}{*}{ Finslerian } & Q1 (DF) & $109.09_{-66.59}^{+68.75},-13.64_{-49.61}^{+20.58}$ & This paper \\
\hline & Q2 (DF) & $296.36_{-103.80}^{+79.05}, 70.00_{-32.58}^{+19.94}$ & This paper \\
\hline & Q1 + Pantheon (DF) & $301.81_{-152.99}^{+56.80},-39.09_{-50.73}^{+17.47}$ & This paper \\
\hline & $\mathrm{Q} 2$ + Pantheon $(\mathrm{DF})$ & $309.09_{-119.77}^{+72.06},-19.09_{-57.63}^{+22.96}$ & This paper \\
\hline & Pantheon (DF) & $298.80_{-118.69}^{+75.31},-23.41_{-57.41}^{+19.26}$ & {$[106]$} \\
\hline & 808Quasars (DF) & $288.92_{-28.80}^{+23.74}, 6.10_{-16.40}^{+16.55}$ & [83] \\
\hline
\end{tabular}

\section{Discussions and conclusions}

In this paper, we tested the cosmic anisotropy in the dipole-modulated $\Lambda \mathrm{CDM}$ model and Finslerian cosmological model with the recently-released quasar sample by using a redshift tomographic method. The quasar sample is divided into two subsets by different cutting redshifts. The results of the two cosmological models are similar except for the dipole amplitude $A_{D}$. The upper limit of dipole amplitude in the Finslerian cosmological model is about a hundred times larger than that in the $\Lambda \mathrm{CDM}$ model. The constraints of matter density $\Omega_{\mathrm{m}}$ are very weak from all the subsets. In the Finslerian cosmological model, the results of matter density $\Omega_{m}$ from the subsets $z \leq 1.0,1.1$ are consistent with ones from the Pantheon sample and CMB, but with large uncertainties. The results of parameters $\beta, \gamma$, and $\delta$ from most subsets $z \leq z_{c u t}$ are consistent within statistical uncertainties, respectively. Though the constraints of matter density $\Omega_{\mathrm{m}}$ are very weak from the subsets $z>z_{\text {cut }}$, the results of the dipole amplitude $A_{D}$ show that quasars 


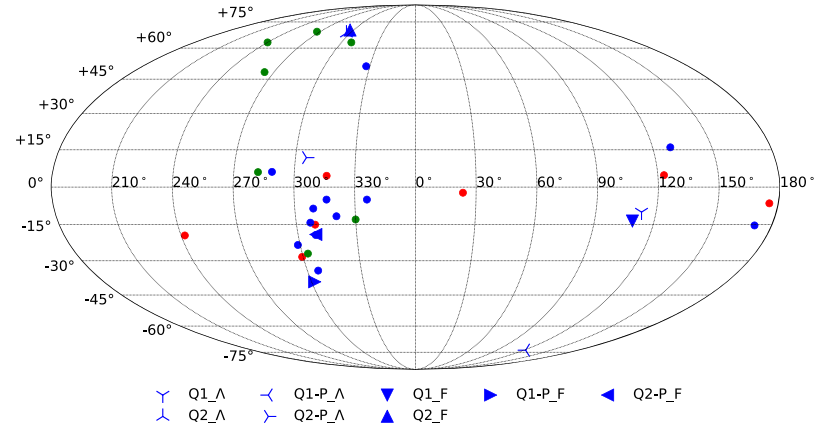

Fig. 6 The distributions of preferred directions derived from different samples in the galactic coordinate system. The blue markers indicate preferred directions obtained by the DF method. The red markers indicate preferred directions obtained by the $\mathrm{HC}$ method. The green markers indicate the other preferred directions. $\Lambda$ indicates the directions in the dipole-modulated $\Lambda \mathrm{CDM}$ model and $\mathrm{F}$ indicates the directions in the Finslerian cosmological model

at a higher redshift range may provide more detailed information about the dipole amplitude $A_{D}$. The dipole directions derived from two subsets divided by different cutting redshifts are consistent within statistical uncertainties except for the subsets Q1 and Q2. In each cosmological model, the dipole directions from the subsets Q1 and Q2 are deviated by $1 \sigma$ level. The Pantheon sample is combined with the two subsets Q1 and Q2 to test the cosmological models. In each cosmological model, the dipole amplitude $A_{D}$ from the combined datasets is very weak. In the dipole-modulated $\Lambda \mathrm{CDM}$ model, the dipole directions from the two combined datasets are far away from each other. For the dataset Q1-P, the dipole direction is $(l, b)=\left(121.82_{-148.87^{\circ}}^{\circ+66.19^{\circ}},-73.64_{-16.36^{\circ}}^{\circ+40.10^{\circ}}\right)$. For the dataset Q2-P, the dipole direction points to $(l, b)=$ $\left(305.47_{-78.58^{\circ}}^{\circ}, 11.82_{-17.39^{\circ}}^{\circ}\right)$. It is worth noting that the dipole direction in the dataset Q1-P is far away from the one $\left((l, b)=\left(306.00_{-125.98^{\circ}}^{\circ+91.94^{\circ}}-23.41_{-54.71^{\circ}}^{\circ}\right)\right)$ in the Pantheon sample [106]. In the Finslerian cosmological model, the dipole directions from the two combined datasets are close to each other. The two directions are also close to the one given by the Pantheon sample in the Finslerian cosmological model.

Our results also show that the inhomogeneous distributions of quasar and Pantheon samples in the sky have significant effects on dipole directions. We need more homogeneous distribution of datasets to search for a convincing cosmic anisotropy. In the future, the surveys such as J-PAS, Euclid, SKA, LSST, and eROSITA, will provide us with larger and more precise observational data of SNe Ia and quasars. Therefore the test of cosmological principle will be more rigorous by employing more precise datasets that have homogeneous distribution in the sky.

Acknowledgements We thank Elisabeta Lusso for providing us with the 2020 compilation of quasars. We thank Zhi-Chao Zhao and Yong
Zhou for helpful discussions. J.-Q.Xia is supported by the National Science Foundation of China under grants No. U1931202 and 12021003 , and the National Key R\&D Program of China No. 2017YFA0402600.

Data Availability Statement This manuscript has no associated data or the data will not be deposited. [Authors' comment: The data underlying this article were provided to us by the authors of arXiv:2008.08586v1].

Open Access This article is licensed under a Creative Commons Attribution 4.0 International License, which permits use, sharing, adaptation, distribution and reproduction in any medium or format, as long as you give appropriate credit to the original author(s) and the source, provide a link to the Creative Commons licence, and indicate if changes were made. The images or other third party material in this article are included in the article's Creative Commons licence, unless indicated otherwise in a credit line to the material. If material is not included in the article's Creative Commons licence and your intended use is not permitted by statutory regulation or exceeds the permitted use, you will need to obtain permission directly from the copyright holder. To view a copy of this licence, visit http://creativecomm ons.org/licenses/by/4.0/.

Funded by $\mathrm{SCOAP}^{3}$.

\section{References}

1. S. Weinberg, Cosmology (OUP, Oxford, 2008)

2. G. Hinshaw et al., Astrophys. J. Suppl. 208, 19 (2013)

3. C.L. Bennett et al., Astrophys. J. Suppl. 208, 20 (2013)

4. N. Aghanim et al., Astron. Astrophys. 641, A1 (2020)

5. M. Tegmark, A. de Oliveira-Costa, A. Hamilton, Phys. Rev. D 68 , 123523 (2003)

6. N. Aghanim et al., Astron. Astrophys. 641, A5 (2020)

7. N. Aghanim et al., Astron. Astrophys. 641, A6 (2020)

8. J.K. Webb et al., Phys. Rev. Lett. 107, 191101 (2011)

9. J.A. King et al., Mon. Not. R. Astron. Soc. 422, 3370 (2012)

10. A. Mariano, L. Perivolaropoulos, Phys. Rev. D 86, 083517 (2012)

11. P. Molaro et al., Astron. Astrophys. 555, A68 (2013)

12. C. Bonvin, R. Durrer, M. Kunz, Phys. Rev. Lett. 96, 191302 (2006)

13. R.-G. Cai, Y.-Z. Ma, B. Tang, Z.-L. Tuo, Phys. Rev. D 87, 123522 (2013)

14. T. Koivisto, D.F. Mota, JCAP 06, 018 (2008)

15. Z. Chang, X. Li, H.-N. Lin, S. Wang, Eur. Phys. J. C 74, 2821 (2014)

16. Z. Chang, H.-N. Lin, Mon. Not. R. Astron. Soc. 446, 2952 (2015)

17. H.-N. Lin, X. Li, Z. Chang, Mon. Not. R. Astron. Soc. 460, 617 (2016)

18. Y. Zhou, Z.-C. Zhao, Z. Chang, Astrophys. J. 847, 86 (2017)

19. Z. Chang, H.-N. Lin, Z.-C. Zhao, Y. Zhou, Chin. Phys. C 42, 115103 (2018)

20. Z. Chang, Y. Zhou, Mon. Not. R. Astron. Soc. 486, 1658 (2019)

21. D. Hutsemekers, R. Cabanac, H. Lamy, D. Sluse, Astron. Astrophys. 441, 915 (2005)

22. V. Pelgrims, D. Hutsemékers, Astron. Astrophys. 590, A53 (2016)

23. P. Tiwari, P. Jain, Astron. Astrophys. 622, A113 (2019)

24. P. Bielewicz, K.M. Gorski, A.J. Banday, Mon. Not. R. Astron. Soc. 355, 1283 (2004)

25. C.J. Copi, D. Huterer, D.J. Schwarz, G.D. Starkman, Mon. Not. R. Astron. Soc. 449, 3458 (2015)

26. Z. Chang, X. Li, S. Wang, Chin. Phys. C 39, 055101 (2015)

27. Y. Akrami et al., Astron. Astrophys. 641, A7 (2020)

28. H.K. Eriksen et al., Astrophys. J. 605, 14 (2004). [Erratum: Astrophys. J. 609, 1198 (2004)]

29. F.K. Hansen, A.J. Banday, K.M. Gorski, Mon. Not. R. Astron. Soc. 354, 641 (2004) 
30. H.K. Eriksen et al., Astrophys. J. Lett. 660, L81 (2007)

31. J. Kim, P. Naselsky, Phys. Rev. D 82, 063002 (2010)

32. J. Kim, P. Naselsky, Astrophys. J. Lett. 714, L265 (2010)

33. A. Gruppuso et al., Mon. Not. R. Astron. Soc. 411, 1445 (2011)

34. H. Liu, A.M. Frejsel, P. Naselsky, JCAP 07, 032 (2013)

35. W. Zhao, Phys. Rev. D 89, 023010 (2014)

36. C. Blake, J. Wall, Nature 416, 150 (2002)

37. A.K. Singal, Astrophys. J. Lett. 742, L23 (2011)

38. C. Gibelyou, D. Huterer, Mon. Not. R. Astron. Soc. 427, 1994 (2012)

39. M. Rubart, D.J. Schwarz, Astron. Astrophys. 555, A117 (2013)

40. P. Tiwari et al., Astropart. Phys. 61, 1 (2014)

41. P. Tiwari, A. Nusser, JCAP 03, 062 (2016)

42. S. Ghosh, P. Jain, Astrophys. J. 843, 13 (2017)

43. J. Colin, R. Mohayaee, M. Rameez, S. Sarkar, Mon. Not. R. Astron. Soc. 471, 1045 (2017)

44. C.A.P. Bengaly, R. Maartens, M.G. Santos, JCAP 04, 031 (2018)

45. P. Tiwari, P.K. Aluri, Astrophys. J. 878, 32 (2019)

46. A.K. Singal, Phys. Rev. D 100, 063501 (2019)

47. N.J. Secrest et al., Astrophys. J. Lett. 908, L51 (2021)

48. T.M. Siewert, M. Schmidt-Rubart, D.J. Schwarz, Astron. Astrophys. 653, A9 (2021)

49. A.K. Singal, Universe 7, 107 (2021)

50. T. Nadolny, R. Durrer, M. Kunz, H. Padmanabhan, (2021)

51. K. Migkas et al., Astron. Astrophys. 649, A151 (2021)

52. K. Migkas, T.H. Reiprich, Astron. Astrophys. 611, A50 (2018)

53. K. Migkas et al., Astron. Astrophys. 636, A15 (2020)

54. L. Shamir, Astrophys. Space Sci. 365, 136 (2020)

55. L. Samushia, B. Ratra, Astrophys. J. 714, 1347 (2010)

56. J.S. Wang, F.Y. Wang, K.S. Cheng, Z.G. Dai, Astron. Astrophys. 585, A68 (2016)

57. F.F. Dirirsa et al., Astrophys. J. 887, 13 (2019)

58. N. Khadka, B. Ratra, Mon. Not. R. Astron. Soc. 499, 391 (2020)

59. S. Cao, J. Ryan, N. Khadka, B. Ratra, Mon. Not. R. Astron. Soc. 501, 1520 (2021)

60. N. Khadka, O. Luongo, M. Muccino, B. Ratra, arXiv:2105.12692 (2021)

61. F. Xu et al., (2020). arXiv:2012.05627

62. F.Y. Wang, Z.G. Dai, E.W. Liang, New Astron. Rev. 67, 1 (2015)

63. H. Wei, JCAP 08, 020 (2010)

64. M.-H. Li, H.-N. Lin, Astron. Astrophys. 582, A111 (2015)

65. M.-H. Li, H.-N. Lin, Astrophys. J. 807, 76 (2015)

66. Z. Chang, X. Li, H.-N. Lin, S. Wang, Mod. Phys. Lett. A 29, 1450067 (2014)

67. D. Mania, B. Ratra, Phys. Lett. B 715, 9 (2012)

68. A.L. González-Morán et al., Mon. Not. R. Astron. Soc. 487, 4669 (2019)

69. S. Cao, J. Ryan, B. Ratra, Mon. Not. R. Astron. Soc. 497, 3191 (2020)

70. R. Chávez et al., Mon. Not. R. Astron. Soc. 442, 3565 (2014)

71. R. Terlevich et al., Mon. Not. R. Astron. Soc. 451, 3001 (2015)

72. J.-J. Wei, X.-F. Wu, F. Melia, Mon. Not. R. Astron. Soc. 463, 1144 (2016)

73. Y. Wu et al., Astrophys. J. 888, 113 (2020)

74. G. Chen, B. Ratra, Astrophys. J. 582, 586 (2003)

75. S. Cao et al., Astron. Astrophys. 606, A15 (2017)

76. J. Ryan, Y. Chen, B. Ratra, Mon. Not. R. Astron. Soc. 488, 3844 (2019)

77. G. Risaliti, E. Lusso, Astrophys. J. 815, 33 (2015)

78. G. Risaliti, E. Lusso, Nat. Astron. 3, 272 (2019)
79. E. Lusso et al., Astron. Astrophys. 642, A150 (2020)

80. N. Khadka, B. Ratra, Mon. Not. R. Astron. Soc. 502, 6140 (2021)

81. N. Khadka, B. Ratra, Mon. Not. R. Astron. Soc. 497, 263 (2020)

82. N. Khadka, B. Ratra, Mon. Not. R. Astron. Soc. 492, 4456 (2020)

83. D. Zhao, J.-Q. Xia, Eur. Phys. J. C 81, 694 (2021)

84. J.-J. Wei, F. Melia, Astrophys. J. 888, 99 (2020)

85. X. Zheng et al., Astrophys. J. 892, 103 (2020)

86. M. Rezaei, S. Pour-Ojaghi, M. Malekjani, Astrophys. J. 900, 70 (2020)

87. J.P. Hu, Y.Y. Wang, F.Y. Wang, Astron. Astrophys. 643, A93 (2020)

88. R. Amanullah et al., Astrophys. J. 716, 712 (2010)

89. N. Suzuki et al., Astrophys. J. 746, 85 (2012)

90. M. Betoule et al., Astron. Astrophys. 568, A22 (2014)

91. D.M. Scolnic et al., Astrophys. J. 859, 101 (2018)

92. I. Antoniou, L. Perivolaropoulos, JCAP 12, 012 (2010)

93. D.J. Schwarz, B. Weinhorst, Astron. Astrophys. 474, 717 (2007)

94. R.-G. Cai, Z.-L. Tuo, JCAP 02, 004 (2012)

95. B. Kalus, D.J. Schwarz, M. Seikel, A. Wiegand, Astron. Astrophys. 553, A56 (2013)

96. X. Yang, F.Y. Wang, Z. Chu, Mon. Not. R. Astron. Soc. 437, 1840 (2014)

97. C.A.P. Bengaly, A. Bernui, J.S. Alcaniz, Astrophys. J. 808, 39 (2015)

98. Z.Q. Sun, F.Y. Wang, Eur. Phys. J. C 79, 783 (2019)

99. J. Colin, R. Mohayaee, S. Sarkar, A. Shafieloo, Mon. Not. R. Astron. Soc. 414, 264 (2011)

100. W. Zhao, P.X. Wu, Y. Zhang, Int. J. Mod. Phys. D 22, 1350060 (2013)

101. H.-N. Lin, S. Wang, Z. Chang, X. Li, Mon. Not. R. Astron. Soc. 456, 1881 (2016)

102. Y.Y. Wang, F.Y. Wang, Mon. Not. R. Astron. Soc. 474, 3516 (2018)

103. Z. Chang, H.-N. Lin, Y. Sang, S. Wang, Mon. Not. R. Astron. Soc. 478, 3633 (2018)

104. Z.Q. Sun, F.Y. Wang, Mon. Not. R. Astron. Soc. 478, 5153 (2018)

105. H.-K. Deng, H. Wei, Eur. Phys. J. C 78, 755 (2018)

106. Z. Chang, D. Zhao, Y. Zhou, Chin. Phys. C 43, 125102 (2019)

107. D. Zhao, Y. Zhou, Z. Chang, Mon. Not. R. Astron. Soc. 486, 5679 (2019)

108. J.S. Wang, F.Y. Wang, Mon. Not. R. Astron. Soc. 443, 1680 (2014)

109. R.-G. Cai, T.-B. Liu, S.-J. Wang, W.-T. Xu, JCAP 09, 016 (2019)

110. D.-C. Qiang, H.-K. Deng, H. Wei, Class. Quantum Gravity 37, $185022(2020)$

111. Y. Avni, H. Tananbaum, Astrophys. J. 305, 83 (1986)

112. X. Li, H.-N. Lin, S. Wang, Z. Chang, Eur. Phys. J. C 75, 181 (2015)

113. D. Foreman-Mackey, D.W. Hogg, D. Lang, J. Goodman, Publ. Astron. Soc. Pac. 125, 306 (2013)

114. H.-K. Deng, H. Wei, Phys. Rev. D 97, 123515 (2018)

115. C.H. Lineweaver et al., Astrophys. J. 470, 38 (1996)

116. M. Frommert, T.A. Enßlin, Mon. Not. R. Astron. Soc. 403, 1739 (2010)

117. D. Hutsemekers et al., ASP Conf. Ser. 449, 441 (2011)

118. R. Watkins, H.A. Feldman, M.J. Hudson, Mon. Not. R. Astron. Soc. 392, 743 (2009)

119. H.A. Feldman, R. Watkins, M.J. Hudson, Mon. Not. R. Astron. Soc. 407, 2328 (2010) 